\title{
Stability Parameter Range of a Tethered Unmanned Aerial Vehicle
}

\author{
Wei He $\mathbb{D}^{1,2}$ and Suxia Zhang $\mathbb{D}^{1,2}$ \\ ${ }^{1}$ Department of Mechanics, School of Mechanical Engineering, Tianjin University, Tianjin 300354, China \\ ${ }^{2}$ Tianjin Key Laboratory of Nonlinear Dynamics and Chaos Control, Tianjin 300354, China \\ Correspondence should be addressed to Suxia Zhang; zhangsux@tju.edu.cn
}

Received 16 September 2021; Revised 11 November 2021; Accepted 6 December 2021; Published 4 January 2022

Academic Editor: Lei Hou

Copyright $\odot 2022$ Wei He and Suxia Zhang. This is an open access article distributed under the Creative Commons Attribution License, which permits unrestricted use, distribution, and reproduction in any medium, provided the original work is properly cited.

\begin{abstract}
In this study, the stability parameter range of a tethered quadrotor unmanned aerial vehicle (UAV) under the action of the transient wind field is numerically analyzed, which can provide a theoretical basis for the design and application of such systems. Three factors affecting the stability of tethered UAV system are determined, namely, cable tension, cable elongation, and UAV vibration velocity, and the corresponding judgment criteria are obtained. Specifically, the priority of the three criteria sequentially decreases. According to these criteria, the stability parameter range of the tethered UAV is examined under the cable parameters such as length, diameter, and elastic modulus and the environmental parameters such as the amplitude and period of the wind field. The results show that for designing the tethered UAV structure, by reducing the length of the tethered cable and increasing its diameter and elastic modulus, the working stability of tethered UAV system can be improved.
\end{abstract}

\section{Introduction}

The velocity of the transient wind field $[1,2]$ rapidly changes. When a tethered unmanned aerial vehicle (UAV) encounters such a wind field during operation, the impact load imposed by the field can affect the working stability of the system. Moreover, the flight control of the system cannot maintain stable control, which may lead to instability and even crash of the UAV. By appropriately designing the mechanical structure parameters, the motion stability of the system can be improved and the energy consumption can be reduced. Therefore, it is crucial to study the structural parameters of tethered UAV system and find the parameter range of stable operation, which can provide theoretical reference for the structural and flight control design of the system.

Tethered UAVs have been extensively examined over recent years. Li et al. [3] used the sliding mode control algorithm to design the flight control system of the tethered UAV system. The flight control system showed certain robustness under the variation of aircraft mass and moment of inertia. Xie [4] explored hover control and trajectory tracking of a tethered quadrotor unmanned helicopter based on the proportional-integral-differential (PID) algorithm and achieved a good control effect. Sandino et al. [5] designed a scheme to connect an unmanned helicopter and the landing point with a tethered cable to ensure a smooth landing of the helicopter. It was found that the tension exerted on the tether had a stabilizing effect on the hovering unmanned helicopter. Marco et al. [6, 7] designed a stabilizing control law for an aerial vehicle, which was physically connected to a ground station through a tethered cable to simultaneously control the UAV flight and maintain cable tension, and they suggested that the tensioned cable can maintain UAV in a stable state. Furthermore, Tognon and Franchi [8] and Tognon et al. [9] designed a nonlinear controller, which could track the position of a tethered UAV with the lower end connected to a mobile platform. Mfiri et al. [10] examined the automatic landing control of a tethered quadrotor UAV at an altitude of $15 \mathrm{~m}$. Tethered UAV system has been widely used in engineering applications, flood control and drought reduction, hydrology and water resource monitoring, water and soil conservation monitoring, river monitoring and supervision, water conservation project monitoring, relay communication, emergency mapping, etc. [11-17]. However, the existing studies 
on the tethered UAV system mainly focus on the relevant control algorithm. To boost the applications of tethered $\mathrm{UAV}$, it is necessary to further explore their dynamic stability from the perspective of mechanical structure.

In the study of tethered aerostat, the dynamic stability of the system plays an important role. Wang et al. [18] established static and dynamic models for a tethered airship. The results showed that the internal tension gradually increased along the tethered cable from the ground and reached its peak at the connection with the airship. Rajani et al. [19] investigated the dynamic stability of a tethered airship under atmospheric disturbance. Yang et al. [20] analyzed the effects of wind power on the stability of the platform in the ground environment. They found that the tethered balloon was relatively stable in the vertical direction, and its stability was mainly determined by the static load of the hot-air balloon and the strength of the cable. Aglietti [21] considered the bending stiffness of the tethered cable to examine the dynamic response of a tethered balloon system at a high altitude under the a transient wind field. The results indicated that the theoretical results might be larger than the actual ones because the material damping of the cable was neglected in the theoretical model. Redi et al. [22] utilized the finite element model to develop an algorithm for simulating the dynamic response of a tethered balloon under transient wind field. The simulation results were found to be in agreement with the theoretical ones, validating the effectiveness of the proposed algorithm in the preliminary evaluation of the technical feasibility of a high-altitude tethered platform system. Kang and Lee [23] established a model to simulate the vibration response of a tethered airship under a transient wind field. The statistical data of the tension, mean value, and standard deviation of the cable indicated that the proposed model could reasonably predict the dynamic behavior of the airborne vehicle. Stanney and Rahn [24] proposed a theoretical model to predict the dynamic response of a tethered airship under a transient wind field, and it was found that the impact load imposed by pulse airflow on the system could cause large tension fluctuation in the cable and even lead to cable failure. For investigating a tethered UAV, we should not only predict the dynamic response of the system through a theoretical model but also establish measures to improve its structural parameters, which can improve its working stability.

In this study, a tethered quadrotor UAV in a hovering state is taken as the research object, and a numerical method is proposed to analyze its stable working parameter range under the action of transient wind fields with different amplitudes and periods. Combined with the basic requirements of stable operation of tethered UAV system in engineering practice, three criteria are obtained for the stable operation. Based on the three criteria, the stable working ranges of different system parameters and environmental parameters are obtained. Accordingly, some measures to improve the system parameter design are established, which can serve as a theoretical reference for the structural and flight control design of tethered UAVs.

\section{Mathematical Model}

Due to the geometrical symmetry of the tethered quadrotor UAV, only the in-plane motion of the system should be considered. Figure 1 shows a schematic of the tethered UAV system, where $V$ is the speed of the wind field. The displacement $u$ of the system is decomposed into components $u_{1}$ and $u_{2}$ along the tangential $e_{1}$ and normal $e_{2}$ directions. $\theta^{0}$ and $\theta$ represent the angles between the tangential direction and the horizontal direction in the initial configuration $R^{0}$ and the dynamic configuration $R^{f}$ of the cable, respectively. The differential equation of motion of the tethered UAV system $[25,26]$ is expressed as follows:

$$
\begin{aligned}
& \rho u_{1, t t}=\left[(P+E A \varepsilon)\left(1+u_{1, s}-K u_{2}\right)\right]_{s}-K(P+E A \varepsilon)\left(u_{2, s}+K u_{1}\right)-\rho g \sin \theta^{0}+F_{t} \\
& \rho u_{2, t t}=\left[(P+E A \varepsilon)\left(u_{2, s}+K u_{1}\right)\right]_{s}+K(P+E A \varepsilon)\left(1+u_{1, s}-K u_{2}\right)-\rho g \cos \theta^{0}+F_{n} \\
& s=0: u_{j}=0, j=1,2 \\
& s=l: m u_{1, t t}(l, t)=F_{L} \sin \left[\theta^{0}(l)+\phi\right]-m g \sin \theta^{0}(l)+F_{D} \cos \left[\theta^{0}(l)+\phi\right]-P_{T}(l, t)\left[1+u_{1, s}(l, t)-K(l) u_{2}(l, t)\right] \\
& m u_{2, t t}(l, t)=F_{L} \cos \left[\theta^{0}(l)+\phi\right]-m g \cos \theta^{0}(l)-F_{D} \sin \left[\theta^{0}(l)+\phi\right]-P_{T}(l, t)\left[u_{2, s}(l, t)+K(l) u_{1}(l, t)\right]
\end{aligned}
$$

where $u_{1, s}$ and $u_{2, s}$ indicate $\partial u_{1} / \partial s$ and $\partial u_{2} / \partial s$, respectively. $\rho$ represents the cable line density. $K$ denotes the static equilibrium curvature of the cable [27]. $P_{T}$ is the internal tension of the cable, expressed as $P_{T}=P+E A \varepsilon$, where $P$ is the static equilibrium tension of the cable [27], $E A$ is the axial stiffness of the cable, and $\varepsilon$ is the dynamic component 


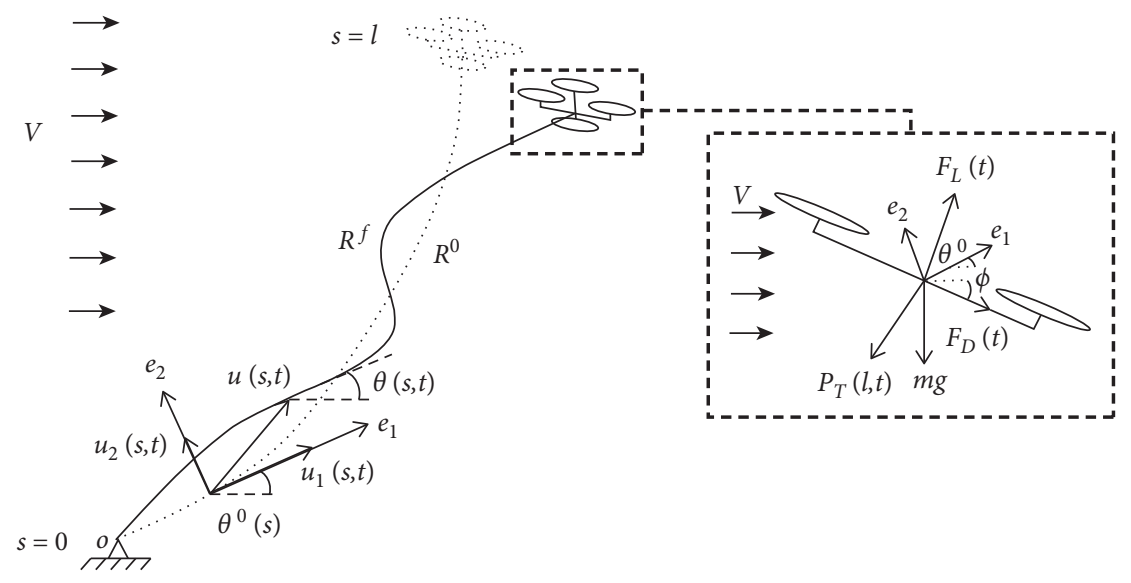

Figure 1: Schematic of the tethered UAV system.

of the Lagrangian strain, which is calculated as

$$
\varepsilon=u_{1, s}-K u_{2}+\frac{1}{2}\left[\left(u_{1, s}-K u_{2}\right)^{2}+\left(u_{2, s}+K u_{1}\right)^{2}\right],
$$

where $F_{t}$ and $F_{n}$ are the tangential and normal wind loads acting on the cable, respectively. They are expressed as follows:

$$
\begin{aligned}
& F_{t}=\frac{1}{2} \rho_{f} C_{d t} d\left(V \cos \theta-u_{1, t}\right)\left|V \cos \theta-u_{1, t}\right|, \\
& F_{n}=\frac{1}{2} \rho_{f} C_{d n} d\left(-V \sin \theta-u_{2, t}\right)\left|-V \sin \theta-u_{2, t}\right|,
\end{aligned}
$$

where $\rho_{f}$ represents the air density; $d$ represents the cable diameter; and $u_{1, t}$ and $u_{2, t}$ represent $\partial u_{1} / \partial t$ and $\partial u_{2} / \partial t$, respectively. $C_{d t}$ and $C_{d n}$ indicate the wind resistance coefficients along with the cable's tangential and normal directions, respectively. According to the experimental verification, $C_{d t}$ is an order of magnitude smaller than $C_{d n}$ [28]. Thus, the tangential wind force $F_{t}$ is ignored in the subsequent calculations.

If the dynamic dip angle of the tethered cable $\theta$ is denoted by $\theta^{0}$ [27], we have

$$
\begin{aligned}
\sin \theta= & {\left[1+u_{1, s}(s, t)-K(s) u_{2}(s, t)\right] \sin \theta^{0} } \\
& +\left[K(s) u_{1}(s, t)+u_{2, s}(s, t)\right] \cos \theta^{0}, \\
\cos \theta= & {\left[1+u_{1, s}(s, t)-K(s) u_{2}(s, t)\right] \cos \theta^{0} } \\
& -\left[K(s) u_{1}(s, t)+u_{2, s}(s, t)\right] \sin \theta^{0} .
\end{aligned}
$$

In this study, it is assumed that the lift forces generated by the four rotors of the UAV are similar, while the rolling motion of the UAV is not considered. The initial rolling angle of the UAV is set as $\phi$ to ensure that it is in an initial equilibrium state.

The air resistance $F_{D}$ of the UAV is expressed as follows:

$$
F_{D}=\frac{1}{2} \rho_{f} C_{D} V_{D}\left|V_{D}\right|
$$

where the relative speed $V_{D}$ of the UAV and the wind field can be obtained as

$$
\begin{aligned}
V_{D}= & {\left[V-u_{1, t}(l, t) \cos \theta(l)+u_{2, t}(l, t) \sin \theta(l)\right] \cos \phi } \\
& -\left[u_{1, t}(l, t) \sin \theta(l)+u_{2, t}(l, t) \cos \theta(l)\right] \sin \phi .
\end{aligned}
$$

The total lift force $F_{L}$ generated by the four rotors $[29,30]$ can be obtained as follows:

$$
F_{L}=\sum_{i=1}^{4} F_{L i}=\sum_{i=1}^{4} 2 \rho_{f} A \sqrt{\frac{k_{1} \Omega_{i}^{2}}{2 \rho_{f} A}} \sqrt{V^{2}+\frac{k_{1} \Omega_{i}^{2}}{2 \rho_{f} A}-2 V \sqrt{\frac{k_{1} \Omega_{i}^{2}}{2 \rho_{f} A} \sin \phi}}
$$

It is well known that the vibration amplitude of the first-order and second-order modes is very large, while the vibration amplitude of the third-order or higher-order modes is extremely small and sometimes imperceptible $[31,32]$. Therefore, the first two modes of the system are considered in both tangential and normal directions. The Galerkin method is used to discretize the dynamic equations (1)-(5) of the system. Furthermore, the first- and second-order ordinary differential equations corresponding to the system dynamics in the tangential and normal directions are obtained [27].

\section{Determination of Stable Working Criteria for the Tethered UAV System}

In practical engineering, tethered UAVs mainly have the following three types of failures. First, the broken cable will make the entire system completely inoperable. Second, when the internal optical fiber of the tethered cable is stretched to exceed the maximum elongation, the normal signal cannot be correctly transmitted between the UAV and ground control system. Third, when the vibration velocity of the UAV exceeds the operating requirements of the equipment carried by the UAV, the system will fail to normally work. From the three failure styles in engineering practice, it is shown that cable tension, cable elongation, and UAV vibration velocity are the most critical and direct factors for system stable work. For a stable operation of the system, the following three criteria must be met: 
(1) The maximum tension on the cable should be less than its breaking strength so that the cable cannot break. This can guarantee the normal power supply of the cable and the continuous flight of the UAV. The SEF-5A-6E composite cable produced by Hefei Ruihe Intelligent Technology Co., Ltd. has been selected in this study, and its breaking strength is $98.067 \mathrm{~N}$.

(2) If the elongation of the cable is less than the allowable elongation, the optical fiber in the composite cable cannot break to ensure the normal transmission of the signal. On the one hand, the reinforcing unit of the SEF-5A-6E composite cable is VICTOR fiber, which has high tensile strength. On the other hand, when the optical and electrical units of the composite cable are twisted, the composite cable has an excess length. For these two reasons, the maximum elongation of the SEF-5A-6E composite cable does not exceed $0.5 \%$.

(3) The movement of the tethered UAV should not exceed the allowable movement range to ensure that the pictures taken are clear and usable. Assuming that the UAV carries a general camera, according to the shooting speed and exposure time, the allowable movement velocity of the UAV can be deduced under the condition of acquiring clear pictures. It is assumed that the focal length of the camera is $300 \mathrm{~mm}$, the length and width of the sensor are 6.4 and $4.8 \mathrm{~mm}$, respectively, and the resolution is $720 \times 1280$. Furthermore, the shutter speed of the camera is considered to be $1 / 50 \mathrm{~s}$. According to the image conversion relationship, to obtain a clear image, the maximum allowable tangential and normal velocities of UAV are 0.374 and $0.184 \mathrm{~m} / \mathrm{s}$, respectively.

The above three criteria are sequentially tested, and all of them must be met, i.e., if the first criterion is not satisfied, the following two criteria need not be evaluated. On this basis, the stable working parameter range of the entire system is evaluated.

\section{Stable Working Range Analysis of a Tethered Quadrotor UAV under Transient Wind Field}

4.1. Determination of the Stable Working Parameter Range Based on the Cable Tension. In this study, the flight control of the UAV is ignored, and the stable working range of a tethered quadrotor UAV is investigated under the transient wind field. The tethered UAV system operates in the Bohai Strait, where the average wind velocity [33] is $5-7 \mathrm{~m} / \mathrm{s}$, and the wind level can reach $10 \mathrm{~m} / \mathrm{s}$. The amplitude and period ranges of the transient wind field are $V_{A}=0-20 \mathrm{~m} / \mathrm{s}$ and $T=0-10$ s, respectively. The specific parameters [34] of the tethered UAV system are listed in Table 1, and these parameters are used to determine the stable working range of the system.

$$
V= \begin{cases}V_{A} \sin (2 \pi t / t) & 0 \leq t \leq T / 2 \\ 0 & t>T / 2\end{cases}
$$

First, the influence of the transient wind field parameters on the tension of the tethered UAV system is analyzed. The wind field period is set as $T=5 \mathrm{~s}$, and the variation in the calculated maximum tension of the cable as a function of the wind field amplitude $\left(V_{A}=0-10 \mathrm{~m} / \mathrm{s}\right)$ is shown in Figure 2(a). It is clear from the figure that as the amplitude of the wind field increases, the tension gradually nonlinearly increases along the cable from bottom to top and reaches the maximum at the connection between the tethered cable and the UAV. Therefore, it is necessary to focus on improving the tensile strength of the cable material at this position. In the figure, the black contour line corresponds to a cable tension of $98.067 \mathrm{~N}$. A vertical dashed line is drawn from the leftmost end of the contour line. It can be seen that the left half of the vertical line indicates the safe area, while the right half denotes the system failure area. When the amplitude of the wind field reaches $6 \mathrm{~m} / \mathrm{s}$, the tension at the upper end of the cable exceeds the breaking strength, and the tethered UAV system fails.

Now, the amplitude of the wind field is set to $V_{A}=5 \mathrm{~m} / \mathrm{s}$, and the tension of the tethered cable under different wind field periods ( $T=0-10 \mathrm{~s})$ is calculated. The results are shown in Figure 2(b), which suggest that the distribution law of cable tension varies with the position. For the wind field periods from 1 to $4 \mathrm{~s}$, the tension of the cable tends to increase first, then decrease, and then again increase. For other wind field periods, the tension gradually increases along the cable from bottom to top. In general, the tension is always within the safe operating range and the cable can safely work. The maximum tension of the cable is concentrated at the upper end of the cable. Therefore, in the design of the tethered cable, the tensile strength of the material in the upper-end area of the cable should be emphasized.

Taking the length, diameter, and elastic modulus of the tethered cable as the main research parameters, the stable working parameter range of the tethered UAV system is investigated based on the parameters in Figure 2. The variation in the maximum tension of the cable as a function of the amplitude and period of the wind field for different cable lengths $(120,140,160,180,200,220$, and $240 \mathrm{~m})$ is shown in Figures 3(a) and 3(b), respectively. In Figure 3(a), the wind field period is $T=5 \mathrm{~s}$; in Figure $3(\mathrm{~b})$, the wind field amplitude is $V_{A}=5 \mathrm{~m} / \mathrm{s}$. Taking breaking tension of $98.067 \mathrm{~N}$ as the standard, the range below the dotted lines in these figures represents the stable working parameter range of the system. For clarity, the corresponding stable ranges are shown in Figures 3(c) and 3(d). In what follows, the stable parameter range is obtained under variable cable diameter and elastic modulus using the same method.

In Figure 3(a), the length of the tethered cable has a minor influence on the tension when the amplitude of the wind field is in the range of $0-3.3 \mathrm{~m} / \mathrm{s}$. However, it has a significant influence when the amplitude exceeds $3.3 \mathrm{~m} / \mathrm{s}$. Moreover, the longer the cable is, the larger the maximum tension is. To reduce the tension of the tethered cable, it is necessary to reduce the length of the cable. In Figure 3(c) corresponding to Figure 3(a), we add a blue dotted line with a wind field amplitude of $7 \mathrm{~m} / \mathrm{s}$, as shown in the following. It can be seen that when the wind field amplitude is lower than 
TABLE 1: Parameters of the tethered UAV system.

\begin{tabular}{lccc}
\hline Parameter & Value & Parameter & Value \\
Line density of the cable $\rho$ & $0.0125 \mathrm{~kg} / \mathrm{m}$ & Cable stiffness $E$ & $1.867 \times 10^{9} \mathrm{~N} / \mathrm{m}$ \\
Cable normal resistance coefficient $C_{d n}$ & $1.2 \mathrm{~m}^{2}$ & Cable diameter $d$ & $0.0034 \mathrm{~m}$ \\
Cable length $l$ & $200 \mathrm{~m}$ & Air density $\rho_{f}$ & $1.29 \mathrm{~kg} / \mathrm{m}^{3}$ \\
UAV quality $m$ & $5.8 \mathrm{~kg}$ & UAV lift coefficient $K_{1}$ & $6.134 \times 10^{-5}$ \\
UAV paddle disc area $A$ & $0.129 \mathrm{~m}^{2}$ & UAV rotor speed $\Omega_{i}$ & $482 \mathrm{rad} / \mathrm{s}$ \\
Air resistance coefficient of UAV $C_{D}$ & $0.064 \mathrm{~m}^{2}$ & UAV inclination $\phi$ & $5^{\circ}$ \\
\hline
\end{tabular}

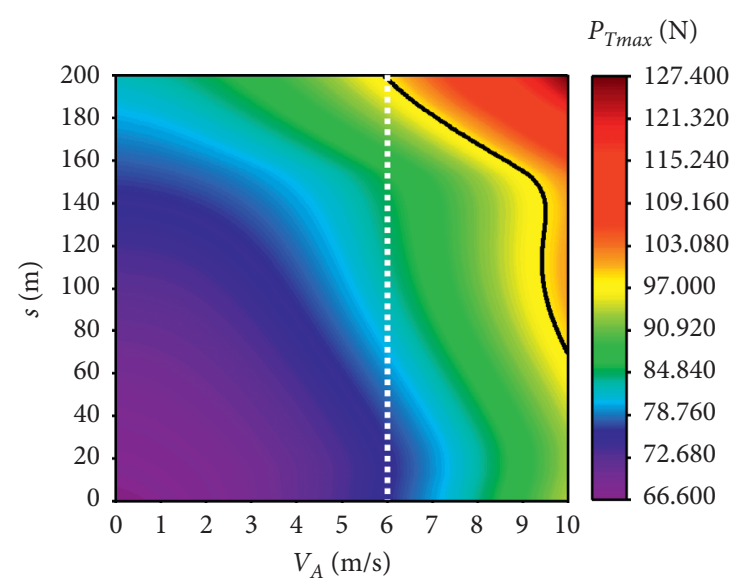

(a)

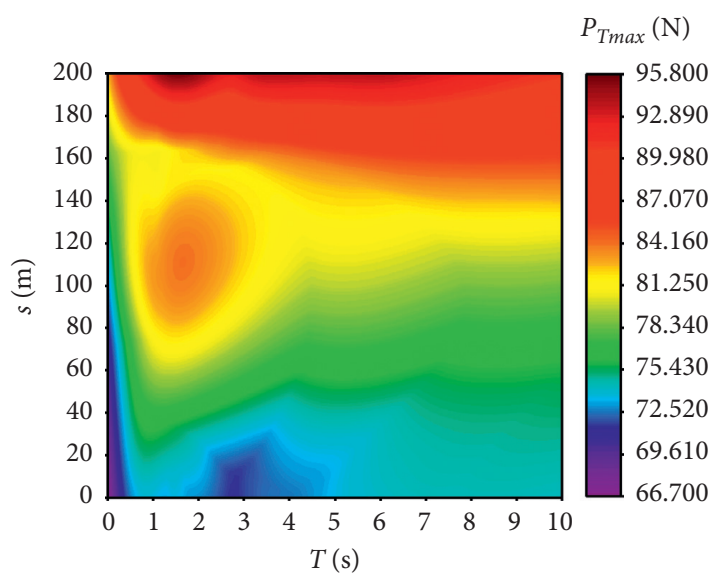

(b)

Figure 2: Maximum tension distribution of the system under transient wind fields with different amplitudes and periods. (a) Maximum tension distribution under transient wind fields of different amplitudes, where the wind field period is $T=5 \mathrm{~s}$. (b) Maximum tension distribution under transient wind fields of different periods, where the wind field amplitude is $V_{A}=5 \mathrm{~m} / \mathrm{s}$.

$7 \mathrm{~m} / \mathrm{s}$, there are still some unstable regions, and as the length of the cable increases, the stable range of the system gradually decreases. Therefore, the length of the cable needs to be reduced. In Figures 3(b) and 3(d), the system is in a safe operating range under all the wind field periods. In this case also, the tension of the cable can be reduced by decreasing its length.

The diameter of the cable directly determines the maximum transmission power, and it also affects the wind load on the cable. When the wind field period is $T=5 \mathrm{~s}$, according to the diameter range of the actual cables, the diameters of the cables are set as 0.0024, 0.0034, 0.0044, and $0.0054 \mathrm{~m}$, and the stability parameter range of the system is obtained, as shown in Figure 4. It can be seen in Figure 4(a) that as the cable diameter increases, the stable working range of the system initially decreases and then increases with a cable diameter of $0.0034 \mathrm{~m}$ as the inflection point. Figure 4(b) shows that except for a small unstable working range within the diameter range of $0.0044-0.0054 \mathrm{~m}$, the system remains stable in all other parameter ranges.

According to the elastic modulus range of the actual tethered cable, the elastic moduli of the cable are set as $6.470 \times 10^{8}, 1.257 \times 10^{9}, 1.867 \times 10^{9}, 2.477 \times 10^{9}, 3.087 \times 10^{9}$, $3.697 \times 10^{9}$, and $4.307 \times 10^{9} \mathrm{~Pa}$, and the stability parameter range of the system is obtained. The results are shown in Figure 5. In Figure 5(a), as the elastic modulus increases, the stability parameter range of the system initially decreases and then slowly increases with the elastic modulus of $2.477 \times 10^{9} \mathrm{~Pa}$ as the inflection point, but the change is not obvious. As the wind field period increases, the maximum tension of the cable remains within the stable working range, as shown in Figure 5(b).

\subsection{Determination of the Stable Working Parameter Range} Based on the Cable Elongation. In the above subsection, the cable tension was considered as the first judgment criterion in determining the stability parameter range, and now the elongation of the tethered cable is taken as the second judgment criterion. Following the same method, the lengths of the tethered cable are selected as 120, 140, 160, 180, 200, 220 , and $240 \mathrm{~m}$ to obtain the stability parameter range of the system. The results are shown in Figure 6. It is clear from Figure 6(a) that the stability range of the system gradually increases with the increase in the cable length; however, the influence of the length of the cable on the elongation is not obvious. In Figure 6(b), the system remains stable in the entire parameter range.

Now, the diameters of the tethered cable are taken as $0.0024,0.0034,0.0044$, and $0.0054 \mathrm{~m}$ to obtain the stable working range of the system, as shown in Figure 7. It is evident from Figure 7 (a) that the stable working range of the system increases with the increase in cable diameter. Figure 7 (b) shows that only a small range with a diameter of $0.0024-0.0034 \mathrm{~m}$ is unstable. 


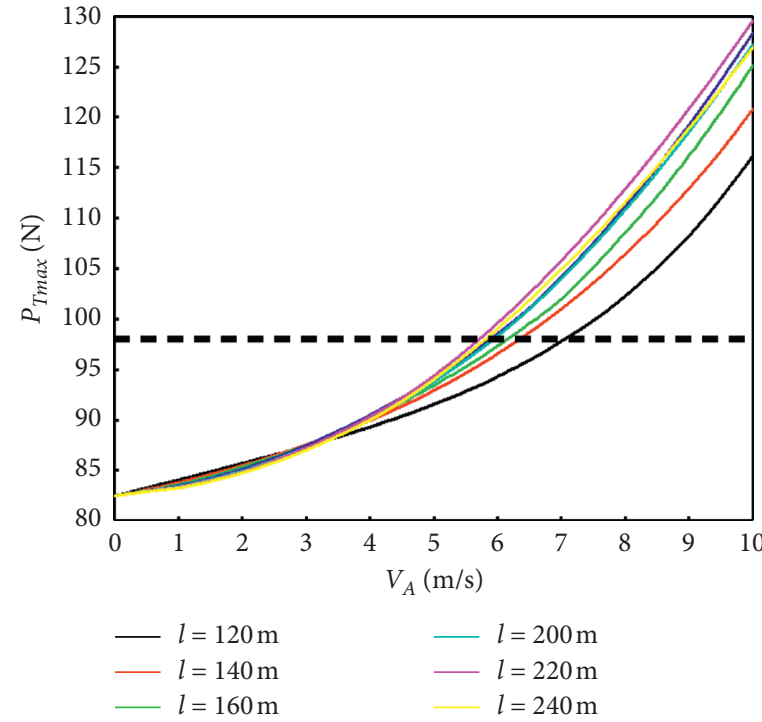

(a)

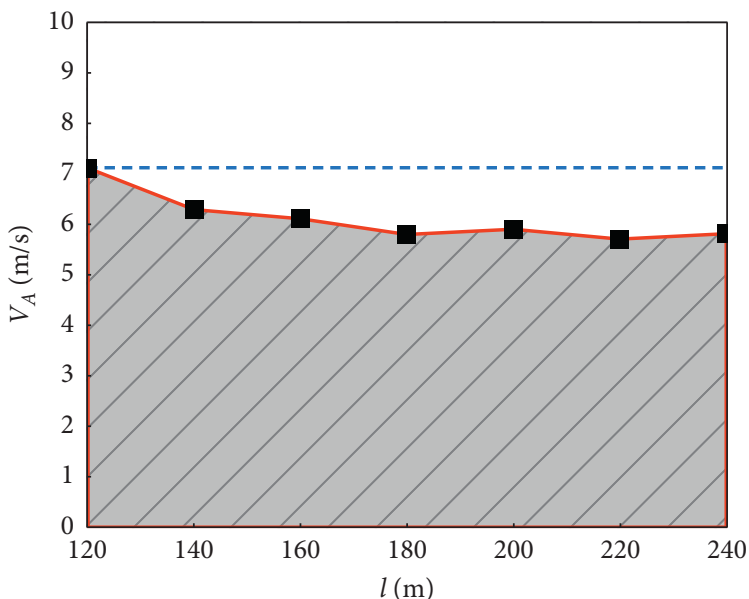

Stable Range

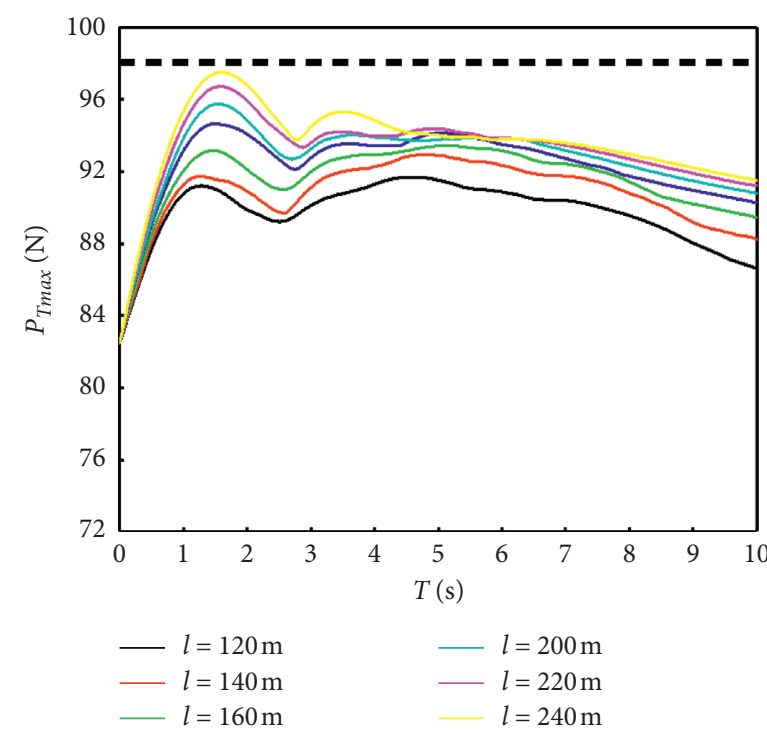

(b)

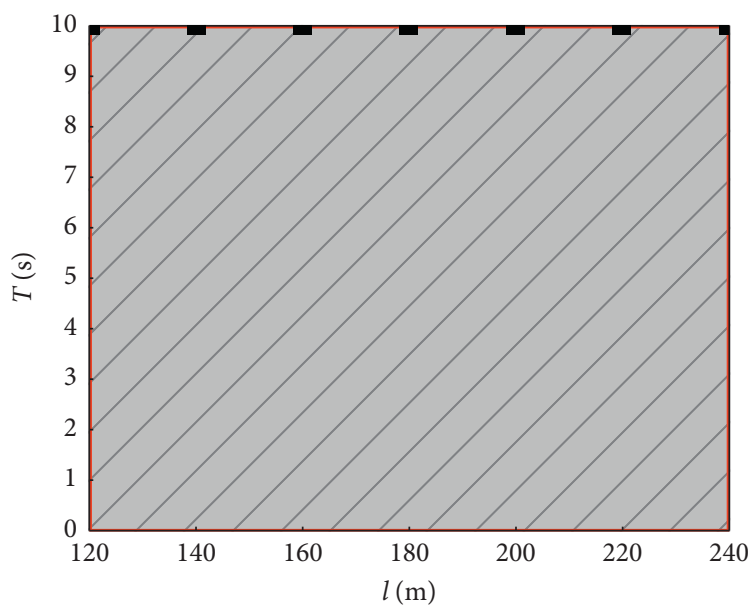

Stable Range

(c)

(d)

FIGURE 3: Stable working range with different cable lengths based on the cable tension. Variation in the maximum tension with (a) wind field amplitude and (b) wind field period under different cable lengths. (c) Stable working range under different wind field amplitudes and cable lengths. (d) Stable working range under different wind field periods and cable lengths.

Next, the elastic moduli of the tethered cable are considered as $6.470 \times 10^{8}, 1.257 \times 10^{9}, 1.867 \times 10^{9}, 2.477 \times 10^{9}$, $3.087 \times 10^{9}, 3.697 \times 10^{9}$, and $4.307 \times 10^{9} \mathrm{~Pa}$ to obtain the stability parameter range of the system, as shown in Figure 8. It is clear from Figures $8(\mathrm{a})$ and $8(\mathrm{~b})$ that when the elastic modulus is greater than $2.477 \times 10^{9}$ and $1.867 \times 10^{9} \mathrm{~Pa}$, respectively, the system is always in a stable state.

\subsection{Determination of the Stable Working Parameter Range} Based on the UAV Velocity. Finally, based on the stability criteria of the tangential and normal velocity of UAV, the stability parameter range of the system is analyzed. The length of the tethered cable is considered as $120,140,160$, $180,200,220$, and $240 \mathrm{~m}$ to obtain the stable working range of the system, as shown in Figure 9. The motion of the UAV is always stable in Figures 9(a) and 9(b). However, in Figures 9(c) and 9(d), the stable working range of UAV decreases with the increase in the cable length.

Now, the diameter of the tethered cable is considered as $0.0024,0.0034,0.0044$, and $0.0054 \mathrm{~m}$ to obtain the stability parameter range of the system, as shown in Figure 10. It can be seen from Figures 10(a)-10(d) that the stability range of the system basically increases with the increase in the cable diameter.

The elastic moduli of the tethered cable are taken as $6.470 \times 10^{8}, 1.257 \times 10^{9}, 1.867 \times 10^{9}, 2.477 \times 10^{9}, 3.087 \times 10^{9}$, $3.697 \times 10^{9}$, and $4.307 \times 10^{9} \mathrm{~Pa}$ to obtain the stability parameter range of the system, as shown in Figure 11. It can be seen from Figures 11(a)-11(d) that as the elastic modulus of 


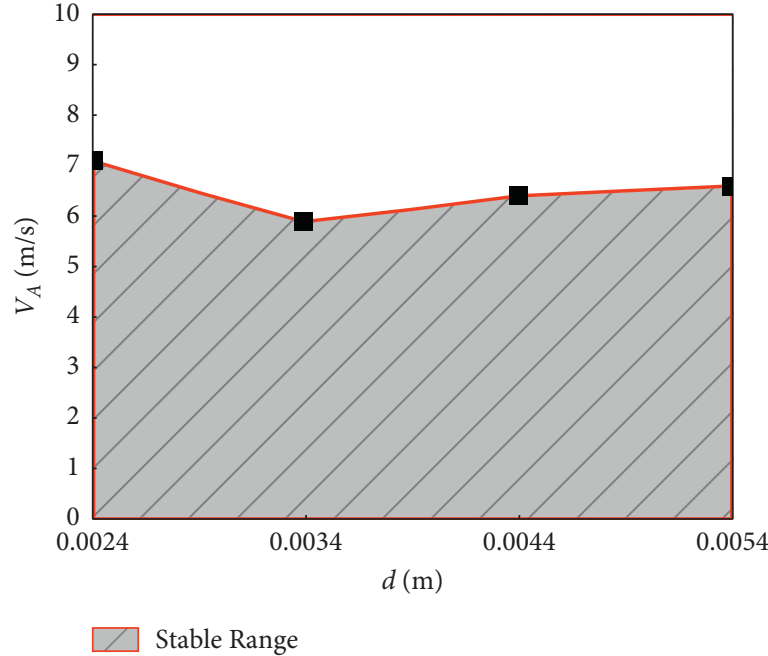

(a)

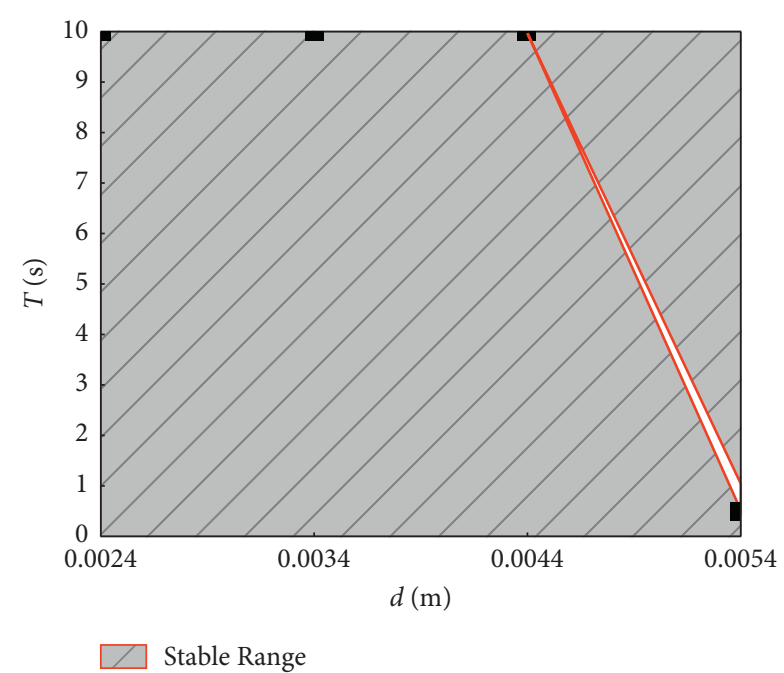

(b)

Figure 4: Stable working range under different cable diameters based on the cable tension. (a) Stable working range under different wind field amplitudes and cable diameters. (b) Stable working range under different wind field periods and cable diameters.

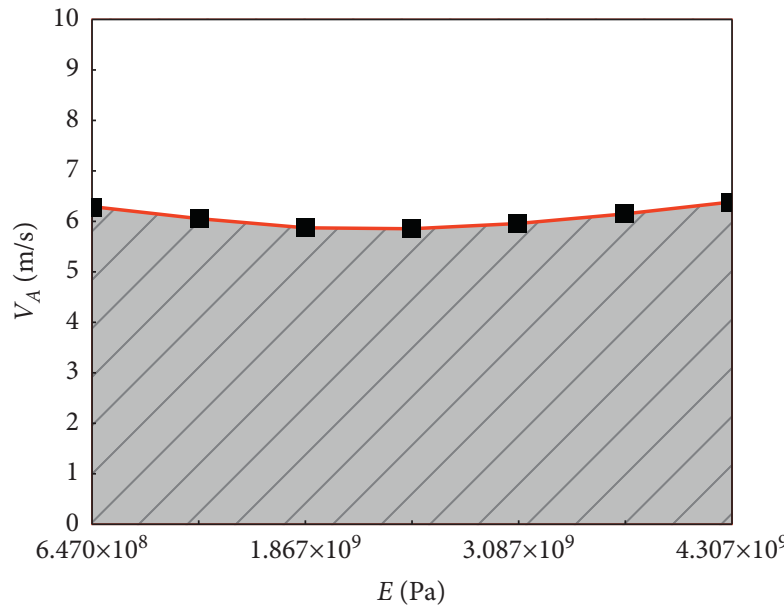

Stable Range

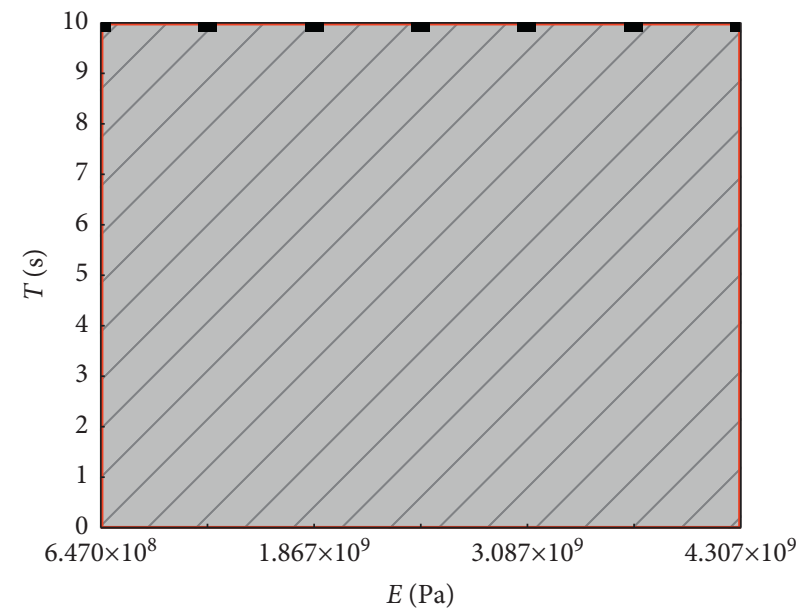

Stable Range

(a)

FIGURE 5: Stable working range under different cable elastic moduli based on the cable tension. (a) Stable working range under different wind field amplitudes and cable elastic moduli. (b) Stable working range under different wind field periods and cable elastic moduli.

the cable increases, the stability parameter range of the system gradually increases.

\section{Engineering Design Suggestions}

We have established three criteria for the stable working condition of tethered UAV system: cable tension, cable elongation, and UAV velocity. The priority of these three criteria sequentially decreases. According to these three criteria, some suggestions for the design of tethered UAV system in engineering practice are as follows:

(1) The cable tension is the first priority based on the fact that the cable should not break. The results suggest that to ensure that the cable tension does not exceed the breaking tension of $98.067 \mathrm{~N}$, the cable length should be reduced, i.e., $l<200 \mathrm{~m}$, and the appropriate cable diameters are $d=0.0024,0.0044$, and $0.0054 \mathrm{~m}$. Moreover, the elastic modulus $E$ of the cable should be smaller than $1.867 \times 10^{9} \mathrm{~Pa}$ or larger than $2.477 \times 10^{9} \mathrm{~Pa}$ to reduce the tension of the cable. By selecting these parameters, the fracture of tethered cable can be avoided.

(2) The elongation of the cable is taken as the second priority to avoid the fracture of the optical fiber inside the cable. To reduce the elongation of the cable and ensure that it does not exceed $0.5 \%$, the cable diameter should be increased, i.e., $d>0.0034 \mathrm{~m}$, and the elastic modulus needs to be increased, i.e., 


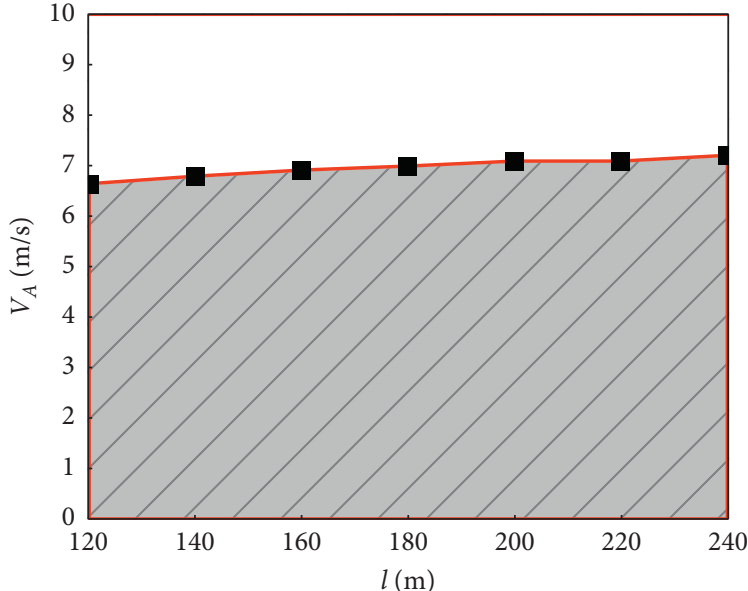

Stable Range

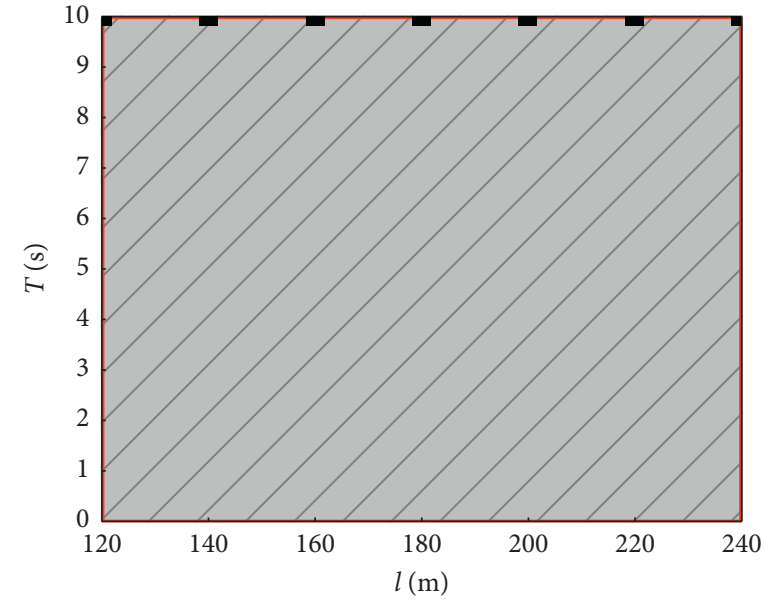

Stable Range

(a)

(b)

FiguRE 6: Stable working range under different cable lengths based on the cable elongation. (a) Stable working range under different wind field amplitudes and cable lengths. (b) Stable working range under different wind field periods and cable lengths.

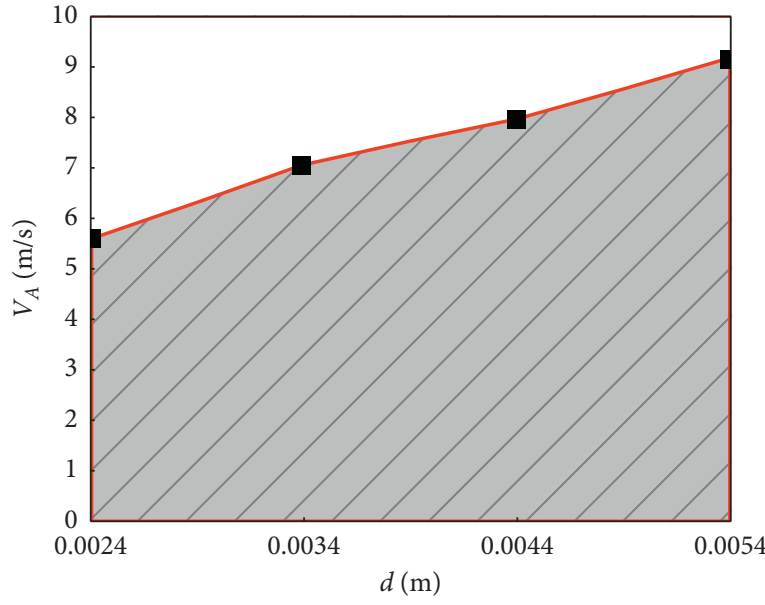

Stable Range

(a)

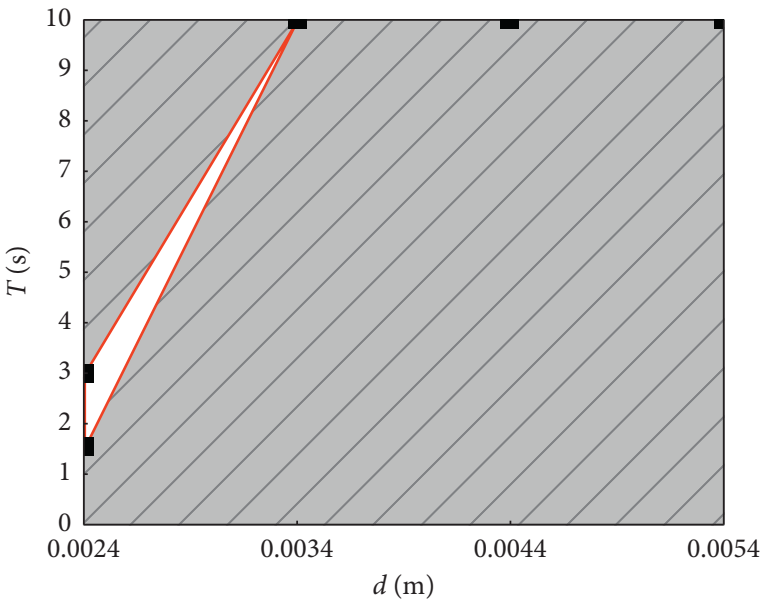

Stable Range

(b)

Figure 7: Stable working range under different cable diameters based on the cable elongation. (a) Stable working range under different wind field amplitudes and cable diameters. (b) Stable working range under different wind field periods and cable diameters.

$E>1.867 \times 10^{9} \mathrm{~Pa}$. The length of the tethered cable has little effect on the elongation, so there is no need to change the length of the cable. By selecting these parameters, the normal data transmission of the optical fiber inside the cable is guaranteed.

(3) The velocity of the UAV is the third priority to ensure that the UAV camera can clearly shoot the target. The results reveal that to reduce the flight velocity of UAV and ensure that the tangential velocity and normal velocity do not exceed 0.374 and
$0.184 \mathrm{~m} / \mathrm{s}$, respectively, it is necessary to reduce the cable length and increase the cable diameter and elastic modulus, i.e., $l<200 \mathrm{~m}, d>0.0034 \mathrm{~m}$, and $E>1.867 \times 10^{9} \mathrm{~Pa}$. By selecting these parameters, the camera can clearly shoot.

Considering the above three working criteria (1), (2), and (3), when designing the tethered UAV system, the length of the tethered cable should be reduced and the diameter and elastic modulus of the cable should be increased to improve the stability of the system. 


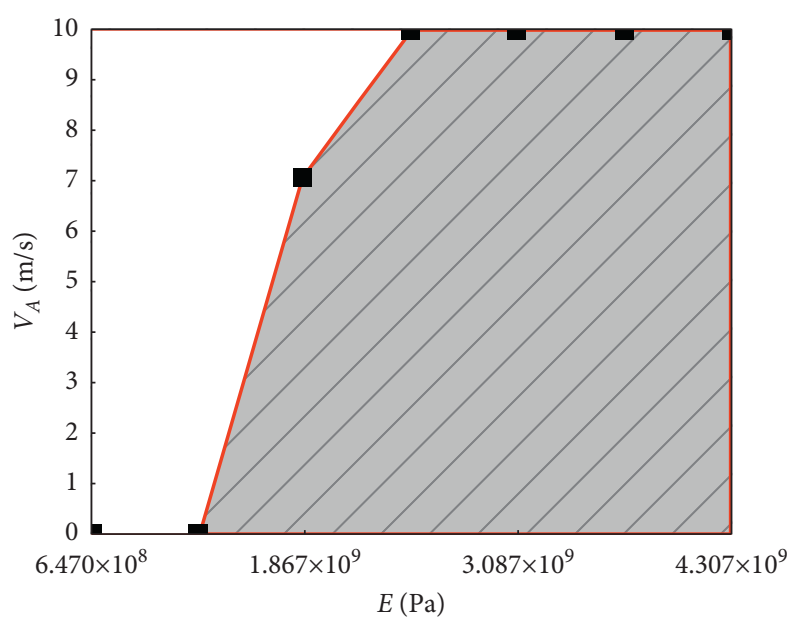

Stable Range

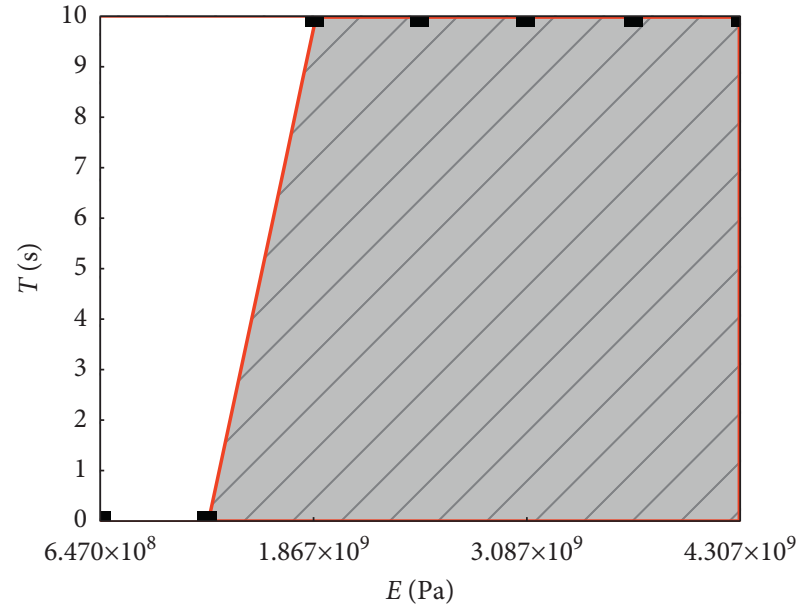

Stable Range

(a)

(b)

FIGURE 8: Stable working range under different cable elastic moduli based on the cable elongation. (a) Stable working range under different wind field amplitudes and cable elastic moduli. (b) Stable working range under different wind field periods and cable elastic moduli.

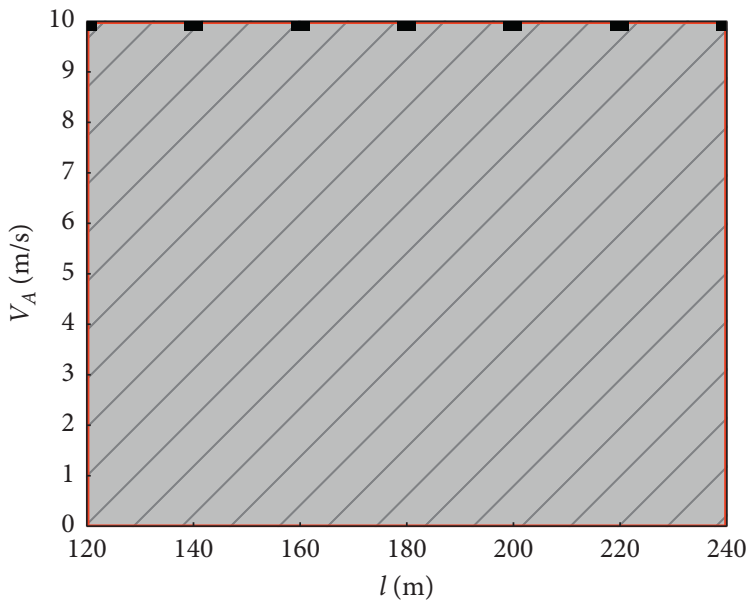

Stable Range

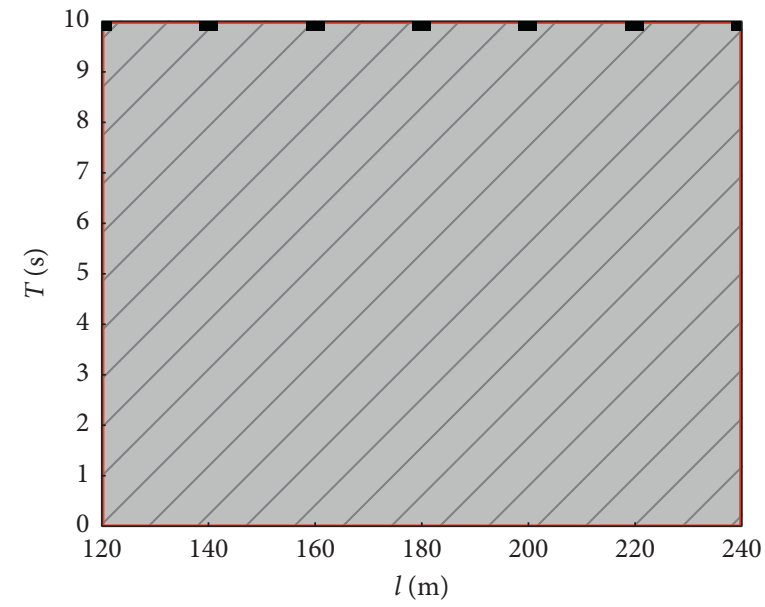

Stable Range

(a)

(b)

Figure 9: Continued. 


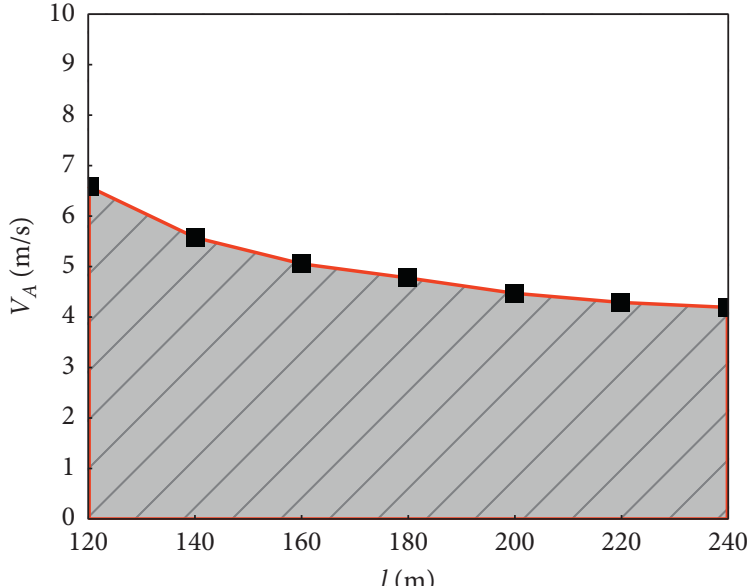

Stable Range

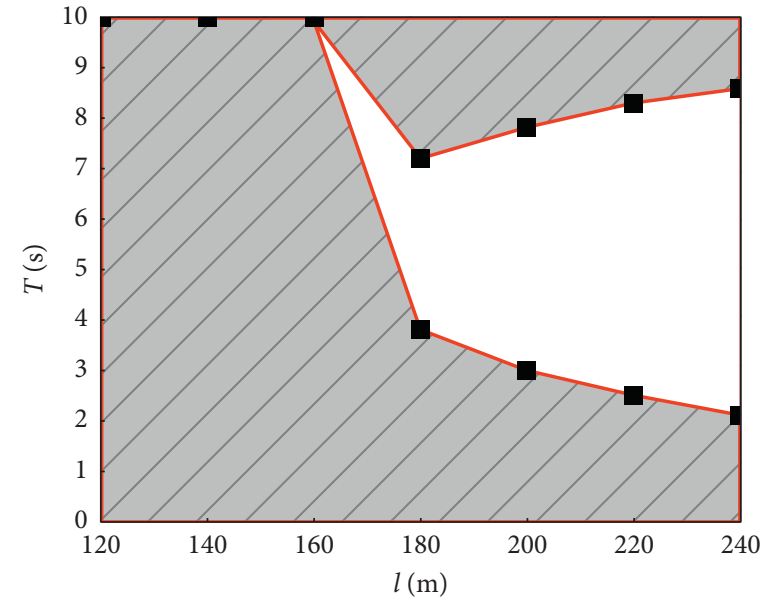

Stable Range

(c)

(d)

FIGURE 9: Stable working range under different cable lengths based on the UAV velocity. (a) Stable working range under different wind field amplitudes and cable lengths based on UAV tangential velocity. (b) Stable working range under different wind field periods and cable lengths based on UAV tangential velocity. (c) Stable working range under different wind field amplitudes and cable lengths based on UAV normal velocity. (d) Stable working range under different wind fields of different periods, and cable lengths based on UAV normal velocity.

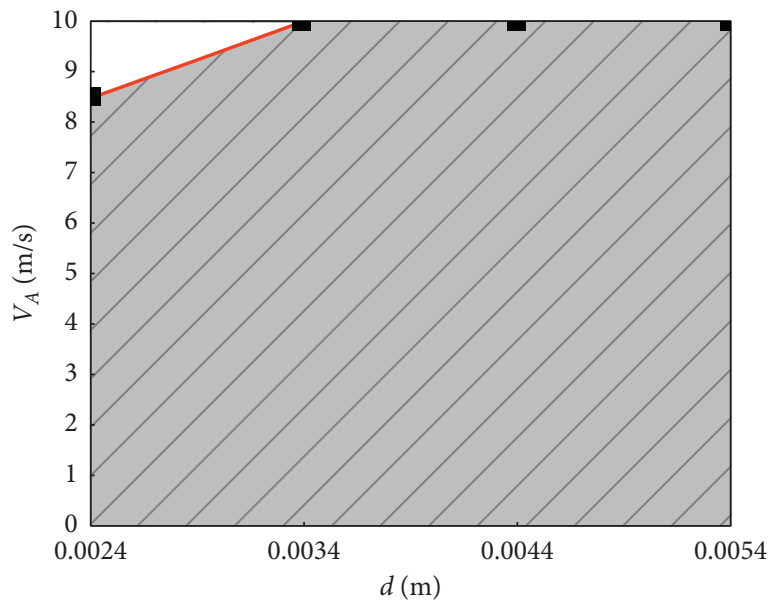

Stable Range

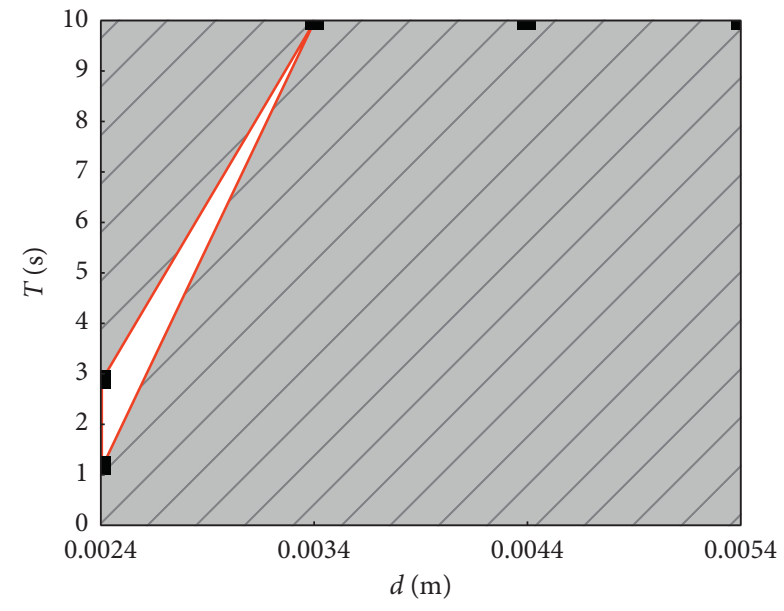

Stable Range

(a)

Figure 10: Continued. 


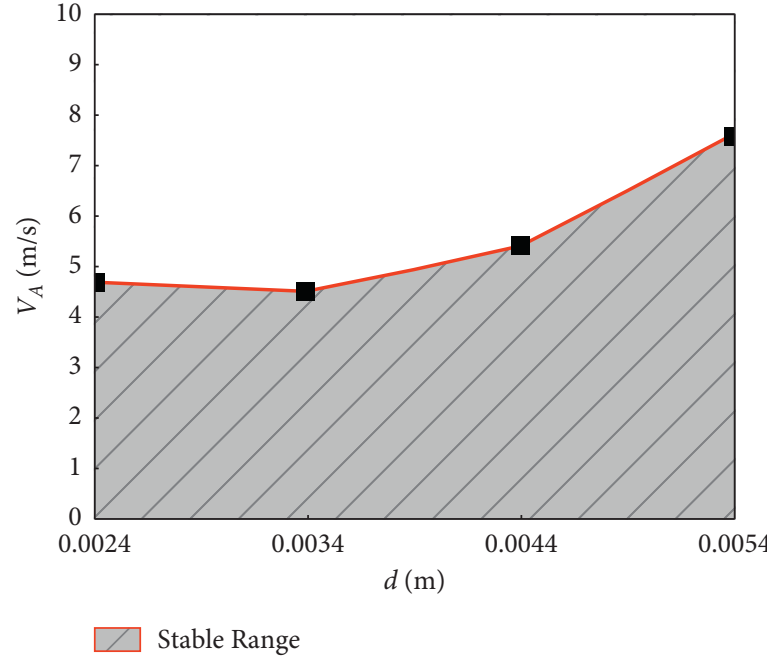

(c)

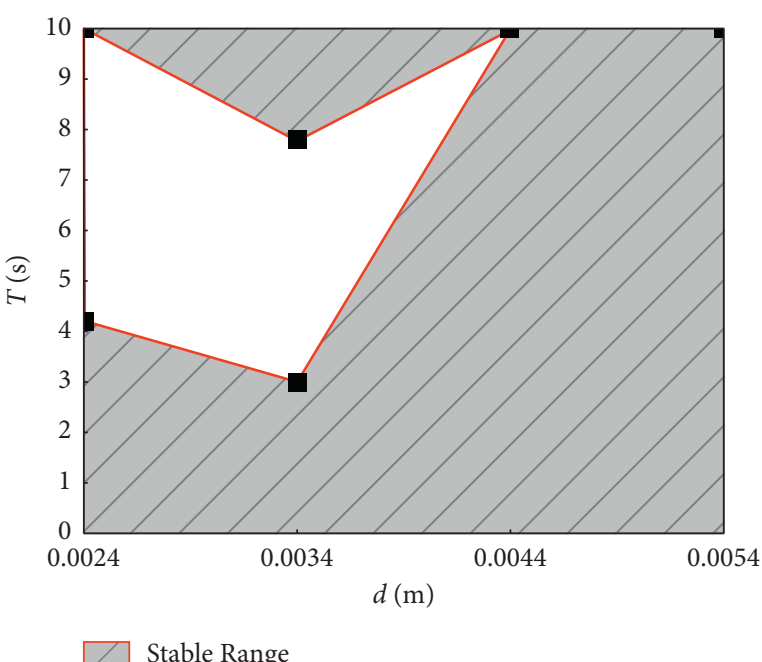

(d)

Figure 10: Stable working range under different cable diameters based on the UAV velocity. (a) Stable working range under different wind field amplitudes and cable diameters based on UAV tangential velocity. (b) Stable working range under different wind field periods and cable diameters based on UAV tangential velocity. (c) Stable working range under different wind field amplitudes and cable diameters based on UAV normal velocity. (d) Stable working range under different wind field periods and cable diameters based on UAV normal velocity.

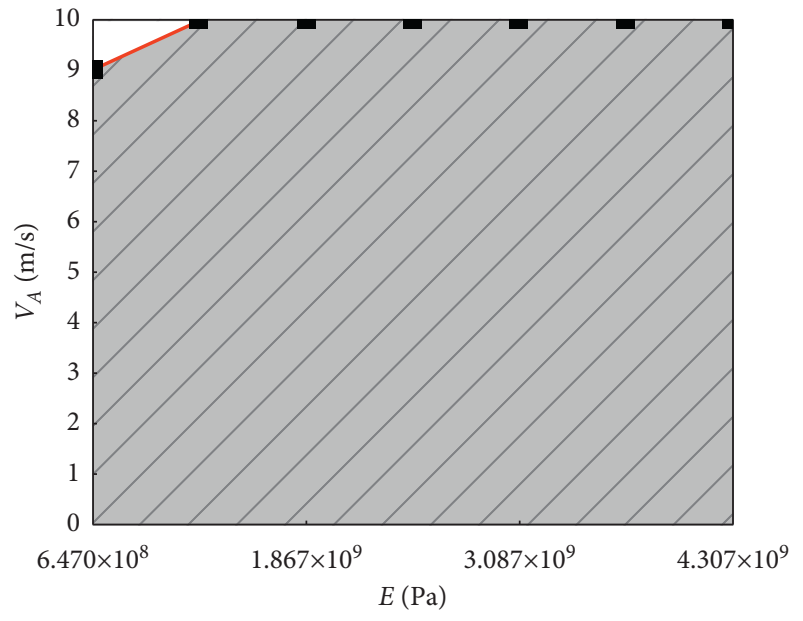

Stable Range

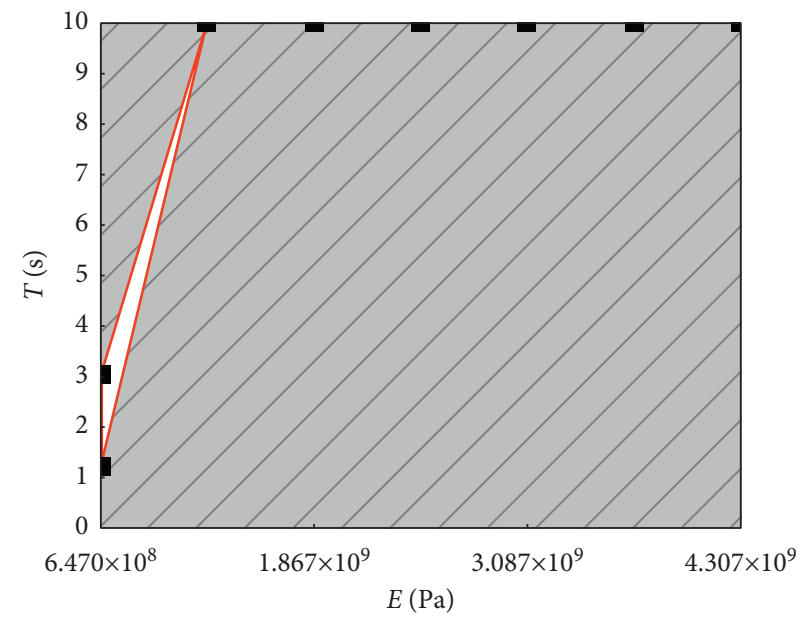

Stable Range

(a)

(b)

Figure 11: Continued. 




Stable Range

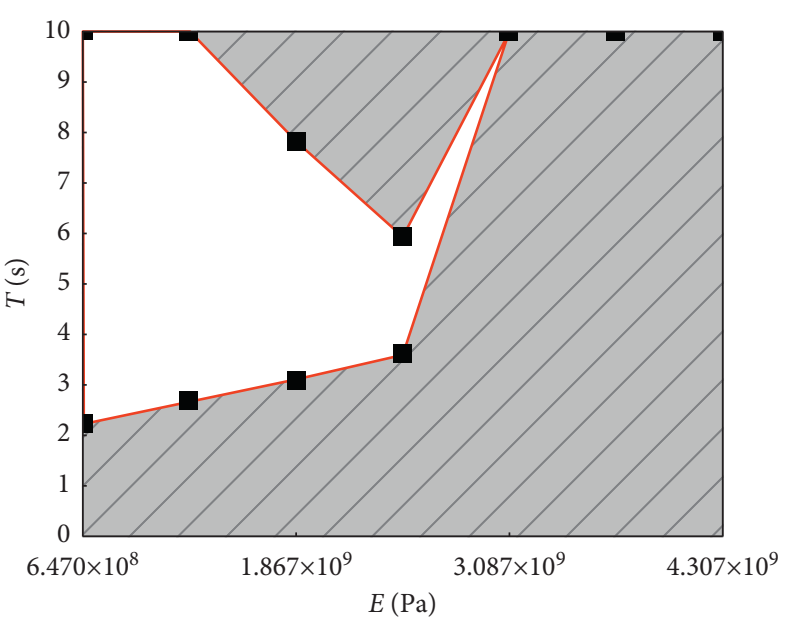

Stable Range

(c)

(d)

FIGURE 11: Stable working range under different cable elastic moduli based on the UAV velocity. (a) Stable working range under different wind field amplitudes and cable elastic moduli based on UAV tangential velocity. (b) Stable working range under different wind field periods and cable elastic moduli based on UAV tangential velocity. (c) Stable working range under different wind field amplitudes and cable elastic moduli based on UAV normal velocity. (d) Stable working range under different wind field periods and cable elastic moduli based on UAV normal velocity.

\section{Conclusions}

The tethered cable can effectively improve the flight time, signal transmission, and flight stability of the UAV. The primary objective of this study was to ensure the safety of tethered cable. Under the dual action of UAV movement and transient wind field, the internal tension of the tethered cable can become extremely large, which causes the cable to break, resulting in the crash of the UAV. Here, combined with the engineering practice, the safe tension value of the tethered cable was identified as the primary criterion to judge the working stability of the system. Second, to ensure that the optical fiber inside the tethered cable allowed normal signal transmission, combined with the practical application of optical fiber, the safe working elongation of optical fiber was specified as the second criterion for the stable operation of the system. Then, to ensure that the UAV camera could capture clear images, combined with the shooting performance of the existing general camera, the stable working velocity of the UAV in tangential and normal directions was specified as the third criterion for the stable operation of the system. By changing the length, diameter, and elastic modulus of tethered cable, the response of tethered UAV system under transient wind field was numerically examined, and the stable parameter ranges of the system under transient wind field with different amplitudes and periods were obtained according to the three proposed stability criteria. Finally, based on the determined stable parameter ranges, some suggestions for the practical engineering design of tethered UAV system were provided. Overall, this study can serve as a theoretical reference for the flight control design and stability improvement of tethered UAVs.

\section{Data Availability}

The data used to support the findings of this study are available from the first author and the corresponding author upon request.

\section{Conflicts of Interest}

The authors declare that there are no conflicts of interest regarding the publication of this paper.

\section{Acknowledgments}

The authors gratefully acknowledge the support of the National Natural Science Foundation of China (51479136), the Project of Tianjin Municipal Transportation Commission (2019-15), the Project of Tianjin Natural Science Foundation (17JCYBJC18700), and the Key Project of Equipment Pre-Research Foundation (61403110101).

\section{References}

[1] Y. Dai, Y. Yuan, S. Song, and Y. Li, "Steady-state and transient wind characteristics of low-rise building roofs with openings in vulnerable areas," Advances in Structural Engineering, vol. 23, no. 11, pp. 2343-2357, 2020.

[2] G. Kristóf, B. Papp, H. Wang, and J. Hang, "Investigation of the flow and dispersion characteristics of repeated orographic structures by assuming transient wind forcing," Journal of Wind Engineering and Industrial Aerodynamics, vol. 197, p. 15, Article ID 104087, 2020.

[3] Z. Li, C. Yang, and Y. Zhu, "Research on sliding mode control algorithm for tethered quadrotor UAV," Electronic Design Engineering, vol. 26, no. 3, pp. 160-164, 2018. 
[4] Q. Xie, "Research on control algorithm for tethered unmanned quadrotor helicopter," Master's thesis in Chinese, Shenyang Aerospace University, Shenyang, Shenbei, China, 2014.

[5] L. A. Sandino, D. Santamaria, and M. Bejar, "Tether-guided landing of unmanned helicopters without GPS sensors," in Proceedings of the IEEE International Conference on Robotics \& Automation, pp. 3096-3101, IEEE, Hong Kong, China, June 2014.

[6] M. M. Nicotra, R. Naldi, and E. Garone, "Taut cable control of a tethered UAV," IFAC Proceedings Volumes, vol. 47, no. 3, pp. 3190-3195, 2014.

[7] M. M. Nicotra, R. Naldi, and E. Garone, "Nonlinear control of a tethered UAV: the taut cable case," Automatica, vol. 78, pp. 174-184, 2017.

[8] M. Tognon and A. Franchi, "Position tracking control for an aerial robot passively tethered to an independently moving platform $* *$ this work has been partially funded by the European union's horizon 2020 research and innovation programme under grant agreement No 644271 AEROARMS," IFAC-PapersOnLine, vol. 50, no. 1, pp. 1069-1074, 2017.

[9] M. Tognon, S. S. Dash, and A. Franchi, "Observer-based control of position and tension for an aerial robot tethered to a moving platform," IEEE Robotics and Automation Letters, vol. 1, no. 2, pp. 732-737, 2016.

[10] J. T. Mfiri, J. Treurnicht, and J. A. A. Engelbrecht, "Automated landing of a tethered quad-rotor UAV with constant winching force," in Proceedings of the 2016 Pattern Recognition Association of South Africa and Robotics and Mechatronics International Conference, pp. 1-6, IEEE, Stellenbosch, South Africa, December 2016.

[11] L. Zeng, R. Li, and Q. Liu, "Discussion on application of flood control, drought relief and disaster reduction based on UAV technology," Harnessing the Huaihe River, vol. 10, pp. 33-34, 2018, (in Chinese).

[12] S. Fu, "The tethered multi-rotor unmanned aerial vehicle and its application to tactical communication," Electronic Techniques of UAV System, vol. 44, no. 4, pp. 14-22, 2018, in Chinese.

[13] X. Qi, A. Ding, W. Nie et al., "Direct measurement of new particle formation based on tethered airship around the top of the planetary boundary layer in eastern China," Atmospheric Environment, vol. 209, pp. 92-101, 2019.

[14] Y. Wang, Q. Li, C. Wang, J. Zhu, and X. Wang, "Tethered UAVs based applications in emergency surveying and mapping," Remote Sensing for Land and Resources, vol. 32, no. 1 , pp. 1-6, 2020, in Chinese.

[15] X. B. Lia, Z. R. Peng, Q. C. Lu et al., "Evaluation of unmanned aerial system in measuring lower tropospheric ozone and fine aerosol particles using portable monitors," Atmospheric Environment, vol. 222, Article ID 117134, 12 pages, 2020.

[16] W. Wang, "Research on establishment of space-air-ground integrated monitoring technology system for air pollution," Master's Thesis in Chinese, Chongqing Industrial and Commercial University, Chongqing, China, 2020.

[17] S. Yang, H. Sui, and X. Yan, "Design and application of high altitude base station communication system based on tethered UAV," Electronics World, vol. 6, pp. 136-137, 2020, in Chinese.

[18] Y. Wang, Y. Jia, Z. Chen, Y. Han, and C. Yang, "Static and dynamic simulation of tethered airship," Spacecraft Recovery \& Remote Sensing, vol. 33, no. 2, pp. 93-99, 2012, in Chinese.
[19] A. Rajani, R. S. Pant, and K. Sudhakar, "Dynamic stability analysis of a tethered aerostat," Journal of Aircraft, vol. 47, no. 5, pp. 1531-1538, 2010.

[20] F. Yang, Q. Zhang, and Z. Feng, "Dynamic model and analysis of tethered balloon system," Computer Simulation, vol. 29, no. 12, pp. 66-70, 2012, in Chinese.

[21] G. S. Aglietti, "Dynamic response of a high-altitude tethered balloon system," Journal of Aircraft, vol. 46, no. 6, pp. 2032-2040, 2009.

[22] S. Redi, G. S. Aglietti, A. R. Tatnall, and T. Markvart, "Dynamic response to turbulence of tethered lighter-than-air platforms," Journal of Aircraft, vol. 48, no. 2, pp. 540-552, 2011.

[23] W. Kang and I. Lee, "Analysis of tethered aerostat response under atmospheric turbulence considering nonlinear cable dynamics," Journal of Aircraft, vol. 46, no. 1, pp. 343-348, 2009.

[24] K. A. Stanney and C. D. Rahn, "Response of a tethered aerostat to simulated turbulence," Communications in Nonlinear Science and Numerical Simulation, vol. 11, no. 6, pp. 759-776, 2006.

[25] N. C. Perkins and C. D. Mote Jr, "Three-dimensional vibration of travelling elastic cables," Journal of Sound and Vibration, vol. 114, no. 2, pp. 325-340, 1987.

[26] W. J. Kim, "Coupled cross-flow and in-line vortex-induced vibration of elastic cable systems," Doctoral Dissertation, The University of Michigan, Ann Arbor, MI, USA, 2001.

[27] W.-J. Kim and N. C. Perkins, "Linear vibration characteristics of cable-buoy systems," Journal of Sound and Vibration, vol. 252, no. 3, pp. 443-456, 2002.

[28] B. M. Sumer and J. Fredsoe, "Hydrodynamics around cylindrical structures," Coastal Engineering, vol. 34, no. 2, p. 313, 1998.

[29] Y.-m. Chen, Y.-1. He, and M.-f. Zhou, "Decentralized PID neural network control for a quadrotor helicopter subjected to wind disturbance," Journal of Central South University, vol. 22, no. 1, pp. 168-179, 2015.

[30] L. E. Munoz and O. Santos, "Robust nonlinear real-time control strategy to stabilize a PVTOL aircraft in crosswind," in Proceedings of the 2010 IEEE/RSJ International Conference on Intelligent Robots and Systems, December 2010.

[31] A. Luongo, D. Zulli, and G. Piccardo, "A linear curved-beam model for the analysis of galloping in suspended cables," Journal of Mechanics of Materials and Structures, vol. 2, no. 4, pp. 675-694, 2007.

[32] A. Zhou, X. Liu, S. Zhang, F. Cui, and P. Liu, "Wind tunnel test of the influence of an interphase spacer on the galloping control of iced eight-bundled conductors," Cold Regions Science and Technology, vol. 155, pp. 354-366, 2018.

[33] "China offshore sea breeze," 2020, http://www.diqiu8.com/ zhongguohaiyang/1880.html in Chinese.

[34] P. G. Ioppo, "The design, modeling and control of an autonomous tethered multirotor UAV," Master's Thesis, University of Stellenbosch, Stellenbosch, South Africa, 2017. 